\title{
Optimization of Annular and Cylindrical Liners for Mixed Exhaust Aeroengines
}

\author{
Thomas R. Law ${ }^{1}$ and Ann P. Dowling ${ }^{2}$ \\ Cambridge University Engineering Department, Trumpington Street, Cambridge, CB2 1PZ, UK
}

\begin{abstract}
The increase in bypass ratios for modern day turbofan engines has led to fan rearward broadband noise emerging as one of the principal aircraft noise sources. The most widespread method for mitigating this noise source is the installation of acoustic liners in the ducting downstream of the fan. Mixed exhaust designs have the potential to be quieter than a three quarter cowl aeroengine due to the additional surface available for liner application. As a result, the Silent Aircraft concept design contains an embedded distributed propulsion system to exploit this opportunity. This paper outlines a suitable method for optimizing the annular and cylindrical liners that make up the exhaust system. To gain maximum benefit, liners should be designed to absorb the modal disturbances that are the most significant for an observer on the ground. The optimization uses an advanced noise footprint cost function to achieve this. Consequently, the liners and the length of the installation have been chosen to meet the aggressive noise target set for the aircraft. In addition, we explore the effect that modal scattering between axial liner junctions can have on the acoustic performance of the system. We find that energy interchange between radial modes will often have a detrimental effect on the overall attenuation, although this can be addressed by selecting the order of the liners in a more considered way. The assumption that scattering between liners can be neglected during optimization to allow design of the individual components therefore appears to be valid in this case. However, subsequent attention has to be directed to the order in which they are placed. Current and next generation mixed exhaust aeroengines will be restricted to smaller liner segment lengths than the Silent Aircraft. This analysis suggests that scattering and hence liner order is likely to more important for these designs.
\end{abstract}

\section{Introduction}

$\mathrm{T}$ HE continuous exponential growth in the air transport industry has led to sustained pressure on airframe and engine manufacturers to address the environmental effects of their products. The Silent Aircraft Initiative (SAI) has demonstrated the aircraft noise benefits that can be achieved if noise is the primary design variable. The overall goal was to generate a credible design that would be virtually inaudible outside of the airport boundary. The aircraft concept is a blended wing with a distributed propulsion system consisting of nine fans embedded in the rear of the airframe and the intakes located on the upper surface (Fig. 1). The high bypass ratio turbofan engine means that one of the major obstacles to success is the rearward propagating fan noise. An extended mixed exhaust installation allows for a substantial amount of acoustic treatment in the exhaust ducting whilst also taking advantage of smaller duct diameters for superior acoustic performance. Conventional engine noise prediction routines are ill-suited to this kind of geometry meaning that a new set of design tools was required.

The modelling of sound propagation in aeroengine ducts as a superposition of 'spinning' modal disturbances is classical. This approach has the advantage of allowing much more insight into the effects of the mean flow, frequency and wall impedance changes when compared to purely numerical methods. Perhaps one of the most important observations is that conventional aeroengine liners are much more effective at attenuating some modal disturbances over others. In general, low order modes are much less readily absorbed than those that are closer to the cut-off criterion. If one considers the ducted acoustic field in terms of ray theory ${ }^{1}$, it is clear that this is because higher order modes propagate at much sharper angles to the lined surfaces and experience many more reflections than a low order mode where the angle is more acute. An in-depth analysis of the eigenvalue solutions in a cylindrical duct by Rienstra ${ }^{2}$ has shown that, in addition to the acoustic solutions, there can be up to four surface

\footnotetext{
${ }^{1}$ Research student, Department of Engineering, AIAA Student Member, tr127@cam.ac.uk

${ }^{2}$ Professor, Department of Engineering, AIAA Member.
} 
waves present. These are so-called as their region of influence is located almost exclusively near to the duct wall. Furthermore, two of these modes may be classified as 'hydrodynamic' given that they only exist in the presence of the mean flow. In fact, one of these modes may be unstable, in a similar way to the classical Helmholtz instability. Such a mode is normally omitted in the acoustic modelling as being an artifact of the boundary condition rather than a true duct mode.

In our preliminary work ${ }^{3}$ on this subject we considered the optimization of the cylindrical mixed exhaust only. It was concluded that liners that bring about the maximum possible reduction in fan broadband acoustic power do not necessarily result in the maximum perceived benefit to the observer. Low order modal disturbances that prove more difficult to attenuate in the duct will often radiate to form the peak in the aircraft noise signature following refraction in the jet shear layer. This will subsequently become the maximum noise level recorded on the ground. The key to being able to optimize individual liner designs within a reasonable timeframe is the assumption that modal scattering due to axial impedance changes along the duct will not have a significant effect on the overall liner performance estimates and radiated sound patterns. This approach has been used elsewhere in the past for cylindrical ducts, for example by $\mathrm{Ko}^{4}$, which has led to the commercially available software package from ESDU ${ }^{5}$. The evaluation of the fully scattered acoustic field is not a trivial problem and is commonly the domain of finite element style calculations ${ }^{6-8}$. Needless to say multimode broadband calculations of this type require a large amount of computer processing time and as a result do not lend themselves to rapid, convenient optimization routines. Elsewhere, another method that has arisen for the mode-matching process between liners is based on the Galerkin Method of Weighted Residuals ${ }^{9}$. This involves matching the pressure and velocity differences across the interfaces via an integration so that the modal constants can be set appropriately. McAlpine et $\mathrm{al}^{10}$ have used this method along with a marching procedure proposed by Cummings ${ }^{11}$ to evaluate the scattering of a blade passing frequency (BPF) tone through an axially segmented inlet liner. This approach is significantly quicker as the problem is manipulated into matrix equations where the arrays are relatively small and the system will converge after only a small number of iterations.

In modal calculations, tonal noise is routinely represented as a small number of related modes linked by the same azimuthal order. Tyler and Sofrin ${ }^{12}$ have shown that rotor-stator interaction tones, that occur at the BPF and associated harmonics will have azimuthal mode orders that are multiples of the blade and vane numbers. At slower engine operating speeds, the first harmonic can be cut-off completely by an 'intelligent choice' of blade and vane numbers. At the same time, the rotor alone pressure field becomes cut-on as the blade tip speed becomes supersonic. Based on the argument that the circumferential phase speed of the rotor will only be supersonic over a small spanwise portion of the blade close to the tip, the BPF tone is frequently modelled using the first radial mode of the azimuthal order equal to the number of

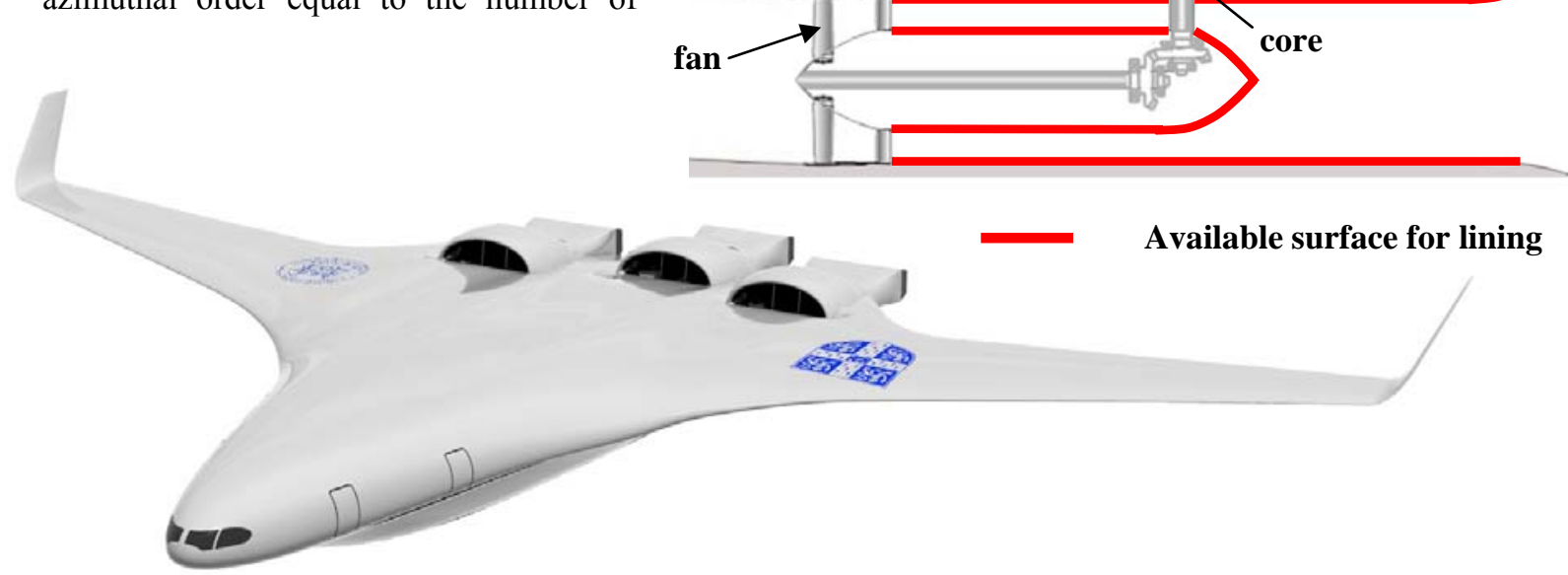

Figure 1. The Silent Aircraft Initiative embedded distributed propulsion system showing a single engine cluster in cross-section with the potential for acoustic lining application. 
blades $^{10}$. This is very important as impedance discontinuities in the axial direction can be used to transfer the tonal energy out of this first radial mode into higher modes so long as the system is azimuthally invariant. Hence, the overall attenuation can be improved as these modes are easier to absorb. Conversely, if there are circumferential discontinuities, energy can be reassigned from a mode that is spinning at high speed to a mode that is significantly slower. In the worse case, one might generate a plane wave which the acoustic treatment can then do little to address. The recent improvements in aircraft noise produced by zero radial splice liner technology ${ }^{8}$ are testament to these principles. In contrast, broadband noise is often modelled as multimodal with equal energy in all cut-on modes. The consequences of scattering are therefore less straightforward to predict as all but the first radial mode in each azimuthal order may scatter its energy into either modes of a lower or higher order. However, given that fan broadband noise has become one of the biggest acoustic challenges associated with modern day designs, it seems important that we should be able to estimate the broadband scattering effects. By causing preferential scattering of tonal noise we may be inadvertently amplifying the broadband signal or there may be additional benefits that have yet to surface. Similarly, it would also be useful to ascertain the validity of optimization methods that neglect the scattering between liner segments.

In this paper, we present an extension to our preliminary work on liner optimization where we now consider the design of all acoustic lining downstream of the fan guide vanes. This includes both annular and cylindrical geometries as we develop a method for mitigation of rearward propagating noise that seeks to gain the maximum possible benefit to the observer. In Section II, we develop the appropriate eigenvalue problems for both the cylindrical and annular geometry and discuss the techniques that we use to solve them. Section III details the development of advanced optimization cost functions that encompass the majority of sound modification mechanisms before the noise reaches the observer. This includes a series of results for the Silent Aircraft design. In Section IV, the scattering of broadband noise in the mixed exhaust is explored using the aforementioned method of weighted residuals. We look to obtain potential attenuation benefits by rearranging liners to get a preferential axial scattering effect.

\section{Annular/Cylindrical Liner Modelling}

The methods used to model acoustic propagation within the annular and cylindrical ducts are an extension of our earlier work on liner optimization ${ }^{3}$.

\section{A. Model Background}

The exhaust system is considered as a combination of annular and cylindrical duct geometries to model the bypass and mixed exhaust ducts respectively.

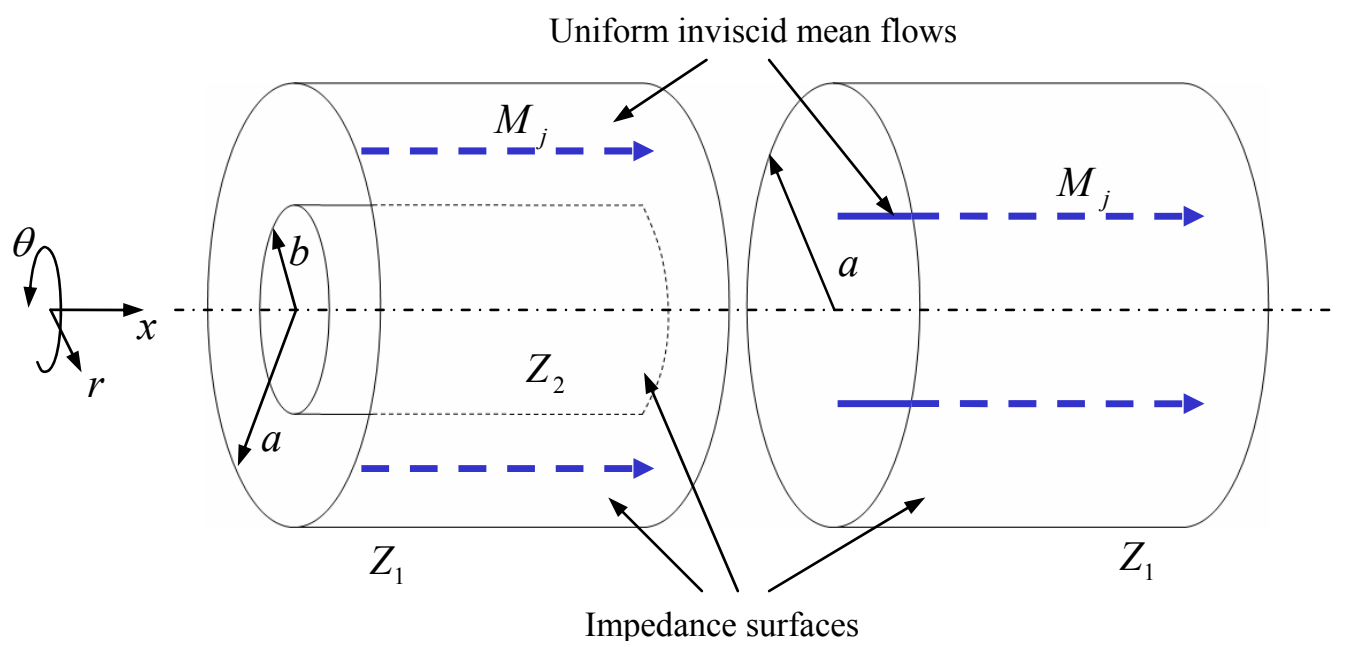

Figure 2. Annular and cylindrical models used to model bypass or mixed exhaust liners respectively.

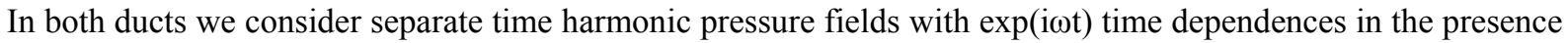
of a uniform inviscid axial flow, Mach number $\mathrm{M}_{\mathrm{j}}$ (specific to the duct being modelled). The external wall is of 
radius a, whilst the internal radius of the annulus is at radius $\mathrm{b}$. For a cylindrical co-ordinate system $(r, \theta, x)$ the acoustic pressure field, $p(r, \theta, x, t)=\hat{p}(r, \theta, x) \exp (i \omega t)$ can be expressed as a modal expansion consisting of upstream (-) and downstream (+) travelling disturbances (Eq. (1)).

$$
\hat{p}(r, \theta, x)=\sum_{n=-\infty}^{\infty} \sum_{m=1}^{\infty} e^{-i n \theta}\left[A_{n m}^{+} e^{-i k_{x, n m}^{+} x} \mathrm{X}_{n m}\left(k_{r, n m}^{+} r\right)+A_{n m}^{-} e^{-i k_{x, n m}^{-} x} \mathrm{X}_{n m}\left(k_{r, n m}^{-} r\right)\right]
$$

Here, $n$ and $m$ are the azimuthal and radial mode orders, $k_{x}$ and $k_{r}$ represent wavenumbers in the axial and radial directions respectively and $A_{n m}$ is an amplitude coefficient. For a cylindrical duct, the radial eigenfunction can be defined as:

$$
\mathrm{X}_{n m}(z)=J_{n}(z)
$$

whereas for an annular duct, it becomes:

$$
\mathrm{X}_{n m}(z)=J_{n}(z)+B_{n m} Y_{n}(z)
$$

$J_{n}$ and $Y_{n}$ denote nth order Bessel functions of the First and Second Kind respectively. The second constant that appears in the annular case, $B_{n m}$, is set by the boundary condition at either duct wall, this is determined in due course. In a uniform axial mean flow, Eq. (1) satisfies the convected wave equation if the axial and radial wavenumbers are related to the free space wavenumber, $\mathrm{k}$, by:

$$
k_{x, n m}^{ \pm}=\frac{-k M_{j} \pm \sqrt{k^{2}-\left(1-M_{j}^{2}\right) k_{r, n m}^{ \pm}}}{1-M_{j}^{2}}
$$

We assume, as is standard, that modes whose corresponding rigid root satisfies the condition below, can be classified as cut-off and will experience substantial rates of decay.

$$
k_{r, n m_{c / o}}>\frac{k}{\sqrt{1-M_{j}^{2}}}
$$

The boundary condition that we impose at the liner surfaces is that the fluid displacement at the wall must match that of the liner (Eq. (6)). This dictates that the radial pressure gradient at the surfaces located at $r=a$ and $b$ satisfies:

$$
\frac{\partial p_{n m}^{ \pm}}{\partial r}=-\frac{i k a}{Z}\left[1-\frac{M_{j} k_{x, n m}^{ \pm} a}{k a}\right]^{2} p_{n m}^{ \pm}
$$

The normalized acoustic impedances are represented by $Z$, where a suffix 1 denotes an outer wall and 2 , an inner wall. For the cylindrical duct, this gives the eigenvalue equation: 


$$
\begin{gathered}
\left(n Z_{1}-C_{n m}^{ \pm}\right) J_{n}\left(k_{r, n m}^{ \pm} a\right)-k_{r, n m}^{ \pm} a Z_{1} J_{n+1}\left(k_{r, n m}^{ \pm} a\right)=0 \\
\mathrm{C}_{\mathrm{nm}}^{ \pm}=-i k a\left[1-M_{j} \frac{k_{x, n m}^{ \pm} a}{k a}\right]^{2}
\end{gathered}
$$

For the annular case, we find that the earlier constant, $B_{n m}$, can be expressed as:

$$
B_{n m}^{ \pm}=\frac{k_{r, n m}^{ \pm} a Z_{1} J_{n+1}\left(k_{r, n m}^{ \pm} a\right)-\left(n Z_{1}-C_{n m}^{ \pm}\right) J_{n}\left(k_{r, n m}^{ \pm} a\right)}{\left(n Z_{1}-C_{n m}^{ \pm}\right) Y_{n}\left(k_{r, n m}^{ \pm} a\right)-k_{r, n m}^{ \pm} a Z_{1} Y_{n+1}\left(k_{r, n m}^{ \pm} a\right)}
$$

The second boundary condition allows us to generate the eigenequation for the annular case.

$\left\lfloor\left(n Z_{1}-C_{n m}^{ \pm}\right) J_{n}\left(k_{r, n m}^{ \pm} a\right)-k_{r, n m}^{ \pm} a Z_{1} J_{n+1}\left(k_{r, n m}^{ \pm} a\right)\right\rfloor\left\lfloor\left(n Z_{2}-C_{n m}^{ \pm} b / a\right) Y_{n}\left(k_{r, n m}^{ \pm} b\right)-k_{r, n m}^{ \pm} b Z_{2} Y_{n+1}\left(k_{r, n m}^{ \pm} b\right)\right\rfloor-$

$\left[\left(n Z_{1}-C_{n m}^{ \pm}\right) Y_{n}\left(k_{r, n m}^{ \pm} a\right)-k_{r, n m}^{ \pm} a Z_{1} Y_{n+1}\left(k_{r, n m}^{ \pm} a\right)\right]\left[\left(n Z_{2}-C_{n m}^{ \pm} b / a\right) J_{n}\left(k_{r, n m}^{ \pm} b\right)-k_{r, n m}^{ \pm} b Z_{2} J_{n+1}\left(k_{r, n m}^{ \pm} b\right)\right]=0$

\section{B. Solution Method and Convergence Schemes}

The eigenvalues can be found by tracking the solutions from the rigid wall to the final value. This method appears to be most successful if we first iterate through impedance values to obtain the no-flow case and then iterate through increasing values of the mean flow. We neglect any instability modes that are found as not being part of the acoustic solution. In a rigid walled duct, the radial wavenumbers, $\kappa_{n m}$ are found by solving either:

$$
J_{n}{ }^{\prime}\left(\kappa_{n m} a\right)=0
$$

or

$$
J_{n}{ }^{\prime}\left(\kappa_{n m} a\right)+B_{n m} Y_{n}^{\prime}\left(\kappa_{n m} a\right)=0
$$

The rigid wall also simplifies the annular constant to:

$$
B_{\mathrm{nm}}=\frac{\kappa_{n m} a J_{n+1}\left(\kappa_{n m} a\right)-n J_{n}\left(\kappa_{n m} a\right)}{n Y_{n}\left(\kappa_{n m} a\right)-\kappa_{n m} a Y_{n+1}\left(\kappa_{n m} a\right)}
$$

As we are interested in modelling broadband noise, we are required to find a substantial number of eigenvalues, particularly at higher frequencies whilst also minimizing the amount of manual input required by the operator. Otherwise, the procedure becomes laborious and of little use as a design tool. The unpredictable nature of the solution domain often necessitates automated root checking and reconvergence with a modified version of Eq. (7) or Eq. (10). Clearly, the annular eigenequation presents a significantly larger challenge to solve due to its increased complexity. This is increasingly the case for low order radial modes as the Bessel Function of the Second Kind will rapidly tend to negative infinity for low arguments. One method that has proved very fruitful for these low order modes is first to converge to the equivalent cylindrical case (this is often a formality). From there, one iterates through increasing values of inner radii, $b$, until the desired annular value is reached. Appendix A contains a proof that the annular eigenvalues do indeed converge to the cylindrical ones at low values of $b / a$. 
Finally, one of the major features that may often prevent the above tracking method from being successful is the branch cut that appears in the presence of flow. This is due to the square root in the evaluation of the axial wavenumber (Eq. (4)). The default branch cut in the expression results in a discontinuity in the complex plane that runs along the real axis from the cut-off criterion to infinity. Regrettably, this will often cut across the root convergence path interfering with the Newton-Raphson scheme. We may change the location of the branch cut and hence create a continuous function in the regions where it is most desirable by modifying the expression for the axial wavenumber under certain conditions:

$$
\begin{gathered}
\xi_{n m}^{ \pm}=\sqrt{k^{2}-\left(1-M_{j}^{2}\right) k_{r, n m}^{ \pm 2}} \\
k_{x, n m}^{ \pm}=\frac{-k M_{j} \pm \xi_{n m}^{ \pm} \operatorname{sgn}\left(-\operatorname{Im}\left(\xi_{n m}^{ \pm}\right)\right)}{1-M_{j}^{2}}
\end{gathered}
$$

The discontinuity now originates from the cut-off criterion on the real axis and extends downwards away from the vast majority of root convergence paths that we are interested in. However, it is important that one exercises care when choosing the sign of the square root to ensure that we get a true solution in later calculations.

\section{Advanced Cost Function Development}

Previous work has suggested that we can obtain appreciable benefits to the observer if we target the peak in the noise radiation pattern as opposed to simply considering levels of residual acoustic power that exit from the exhaust nozzle. The original cost functions were very simple and have now advanced to include many more features.

\section{A. Individual Liner Optimization}

When optimizing an individual liner design, there are a number of material parameters to vary. However for a single degree of freedom system, the main ones are the facing sheet porosity and the backing cell depth. Other ones such as facing sheet thickness and perforate hole diameter will typically improve as they are reduced and are therefore set by material and manufacturing restrictions rather than performance estimates. As a result, we have set all other values to be typical of existing industry designs and are considering changes in the main two only. For the scope of this investigation we will be considering annular liner segments that have identical impedances on both the inner and outer walls, i.e. $Z_{l}=Z_{2}$. The impedance boundary condition is based on actual liner parameters to ensure a physically realisable result ${ }^{13,14}$. The performance of any particular acoustic liner will vary considerably over the frequencies of interest. Fig 3 shows a typical liner performance over a range of frequencies. A common technique when considering broadband noise is to evaluate the performance at third octave centre frequencies. For liners with larger cell depths (designed for lower frequencies) this is quite appropriate, however the performance at smaller cell depths is likely to vary considerably across the larger third octave bands. This means that one may choose a liner design that may not be quite optimal for the conditions under which it is operating. To avoid this problem, we examine the liner performance over a small band centered on the principle liner design frequency. By this term, we are referring to the frequency where the cell depth is equivalent to a quarter of the wavelength. This will not be exact as there are other effects that influence the liner reactance but this is by far the dominant one. For a unit length of liner we can estimate the equal energy per mode attenuation, $\Delta$, using Eq. (16). The limits $n_{\max }$ and $m_{n, \max }$ denote the maximum azimuthal and radial modes that occur prior to cut-off as defined by Eq. (5).

$$
\Delta=\frac{\sum_{n=-n_{\max }}^{n_{\max }} m_{n, \max }}{\sum_{n=-n_{\max }}^{n_{\max }} \sum_{m=1}^{m_{n, \max }} e^{4 \operatorname{Im}\left(k_{x, n m}^{+} a\right)}}
$$




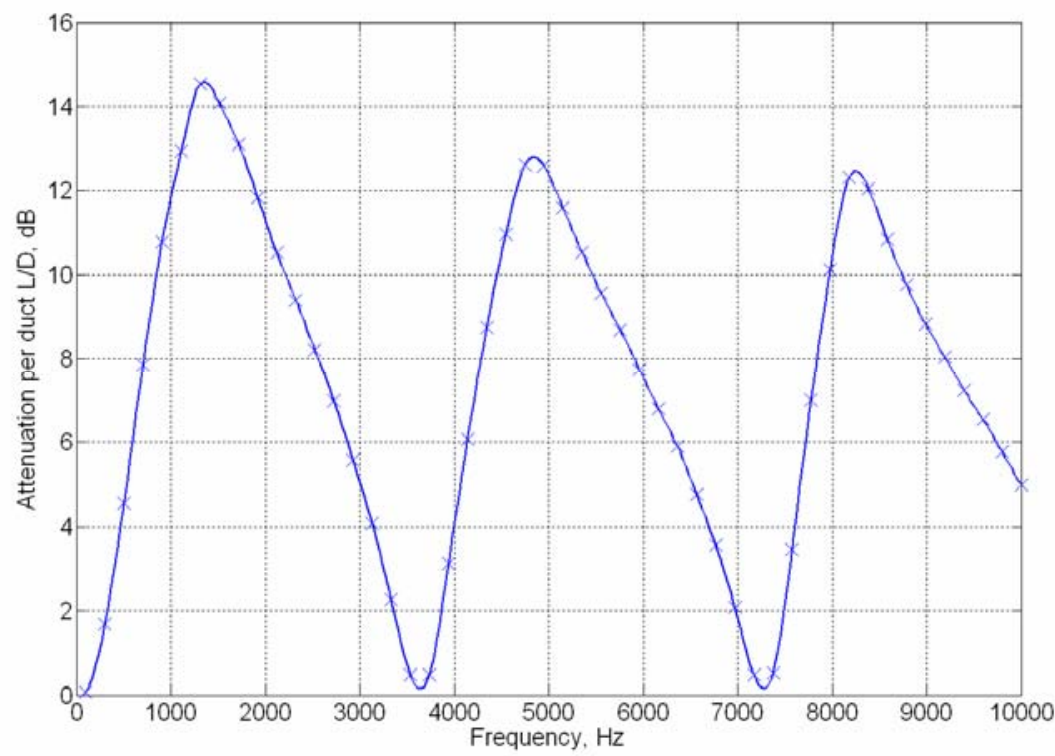

Figure 3. Performance of a single layer cylindrical liner, cell depth $50 \mathrm{~mm}$, porosity $10 \%$ in the presence of a mean flow of Mach number 0.47

We evaluate this Eq. (16) at a number of points around the principle design frequency to allow the performance curve to be adequately formed, for example, ten points over a band width of $400 \mathrm{~Hz}$. If we integrate this quantity over this frequency band we have a suitable measure of liner performance to compare liners of equal cell depth but varying porosity. Then, for a given cell depth we compute this integral for a range of porosities that will encompass the point of optimum damping. Based on experience this will normally occur at a porosity within the range $5-20 \%$ and will depend heavily on the speed of the mean flow in the duct. A simple routine to find the design value is then executed. In general, the metric will vary in a similar way to that of Fig.4.

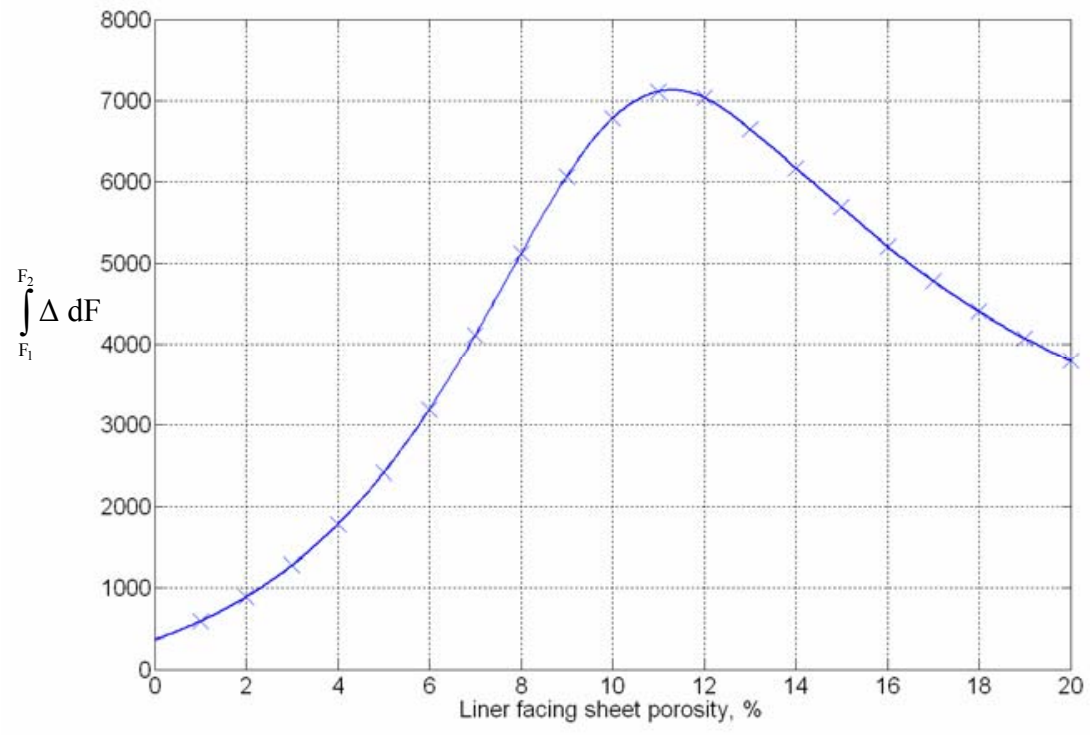

Figure 4. Variation of the performance metric as porosity is varied for the liner of Fig. 3. 


\section{B. Bypass Liner Length Optimization}

Given that the Silent Aircraft has an extended common nozzle design, it becomes pointless to attempt to gauge the effect to the observer of the sound absorption in the bypass duct. Especially given that the liner design and hence performance further downstream is going to depend on the design chosen here. Therefore, the most suitable cost function here would appear to be the residual acoustic power that exits the duct.

The acoustic power associated with any particular mode is given by integrating the axial intensity, $I_{x}$, over the duct cross-sectional area:

$$
W_{n m}^{ \pm}=2 \pi \int_{b}^{a} I_{x, n m}^{ \pm} r d r
$$

An expression for the acoustic intensity in the presence of a mean flow has been given by Morfey ${ }^{15}$ and can be expressed as:

$$
\begin{gathered}
I_{x, n m}^{ \pm}=\frac{\Omega_{n m}^{ \pm}}{2 \pi}\left|p_{n m}^{ \pm}\right|^{2} \\
\Omega_{n m}^{ \pm}=\frac{\pi}{\rho_{j} c_{j}}\left[\left(1+M_{j}^{2}\right) \operatorname{Re}\left(\eta_{n m}^{ \pm}\right)+M_{j}\left(1+\left|\eta_{n m}^{ \pm}\right|^{2}\right)\right] \\
\eta_{n m}^{ \pm}=\frac{k_{x, n m}^{ \pm} a}{\left(k a-M_{j} k_{x, n m}^{ \pm} a\right)}
\end{gathered}
$$

Here $\rho_{j}$ and $c_{j}$ represent the density and sonic speed of the mean flow. The integral can thus be evaluated to give an expression for the acoustic power of a particular mode. If we have the rigid wall condition we obtain the following result for an annular duct by exploiting the recurrence relations of the Bessel Functions ${ }^{16}$ :

$$
W_{n m}^{ \pm}=\frac{a^{2}}{2}\left|A_{n m}^{ \pm}\right|^{2} \Omega_{n m}^{ \pm}\left\{\left[1-\left(\frac{n}{\kappa_{n m} a}\right)^{2}\right]\left|\mathrm{X}_{n m}\left(\kappa_{n m} a\right)\right|^{2}-(b / a)^{2}\left[1-\left(\frac{n}{\kappa_{n m} b}\right)^{2}\right]\left|\mathrm{X}_{n m}\left(\kappa_{n m} b\right)\right|^{2}\right\}
$$

The equivalent result for a cylinder will become useful later. In this case the lower limit of the integral is zero and we obtain:

$$
W_{n m}^{ \pm}=\frac{a^{2}}{2}\left|A_{n m}^{ \pm}\right|^{2} \Omega_{\mathrm{nm}}^{ \pm}\left[1-\left(\frac{n}{\kappa_{n m} a}\right)^{2}\right]\left|\mathrm{X}_{n m}\left(\kappa_{n m} a\right)\right|^{2}
$$

If we can estimate the levels of rearward propagating acoustic power produced by the fan, we use Eq. (21) to set modal amplitudes appropriately at the start of the bypass duct. The inherent assumption being that we can model broadband noise by assuming equal energy in all cut-on propagating modes, which is a widely accepted technique. At this stage, we neglect scattering due to surface impedance changes between liners to allow the optimization to proceed: these effects are evaluated later. As a result we can estimate the amplitude squared of each downstream propagating mode by accounting for the axial decay rates due to the presence of $q_{\max }$ different liners using Eq. (23). 


$$
\left|A_{n m_{\text {OUT }}}^{+}\right|^{2}=\left|A_{n m_{I N}}^{+}\right|^{2} \prod_{q=1}^{q_{\max }} e^{\frac{4 L_{q}}{D} \operatorname{Im}\left(k_{x, n m}^{+} a\right)}
$$

In all this work we have used an industry standard code to estimate fan broadband noise in the twenty four thirdoctave bands that range from centre frequencies of $50 \mathrm{~Hz}$ to $10 \mathrm{kHz}$. The spectrum of interest will now be modelled as these centre frequencies. Although we can estimate the power at each frequency reaching the end of the bypass duct, liner optimization necessitates that we have a scalar measure of liner performance. This will be the integrated A-weighted residual acoustic power level (PWL). The following routine will then provide a suitable liner design.

1. Choose a suitable selection of cell depths that are going to be collectively capable of addressing the input noise spectrum.

2. Optimize each one individually using the method outlined in Section B.

3. Evaluate the eigenvalues for each liner for the modes cut-on at each of the twenty four third octave frequencies.

4. Express the metric as a simple function of liner L/D ratios based on Eq. (23).

5. Run a second optimization loop to find the best combination of lengths against the new cost function.

This routine has the distinct advantage that unsuitable (or less effective) liners will have the corresponding L/D ratio set to zero.

\section{Mixed Exhaust Liner Length Optimization}

Once a design has been established for the bypass, we may move onto the mixed exhaust downstream. Modal amplitudes are now allocated based on Eq. (22). However, given that we have estimated modal attenuation information from the previous duct, we do not assume equal energy per cut-on mode at the start of this duct. Instead, we assume that the energy in each azimuthal order is conserved from the bypass, given that the entire system has rotational symmetry, we would not expect there to be any scattering azimuthally. The energy is then distributed equally amongst the radial modes for any particular value of $\mathrm{n}$. In addition, all acoustic energy that reached the end of the bypass is assumed to be transferred to the downstream duct. This is most likely a conservative approach.

As this duct is the final part of the engine before the noise exits to the atmosphere, the cost function that we develop here is going to be the maximum perceived noise outside of the airport boundary. For a multiple segment liner, one can estimate the amplitudes of the modal disturbances that reach the end of the final nozzle at the same centre frequencies using Eq. (23) in the same way as the ducting upstream. We can then estimate the sound pressure level associated with a particular frequency at a distance, $R$, and polar angle, $\psi$, from the engine exhaust line using Eq. (24). The quantity $D_{n m}$ is a modal directivity factor that defines its radiation from the nozzle. We can compute good estimates of this using the Wiener-Hopf solution for an unflanged semi-infinite cylindrical duct, see Munt ${ }^{17}$ and Gabard and Astley ${ }^{18}$.

$$
\operatorname{SPL}(\psi, R)=10 \log _{10}\left[\sum_{n=-n_{\max }}^{n_{\max }} \sum_{m=1}^{m_{\max }^{n}}\left|A_{n m_{E X I T}}^{+} D_{n m}(\psi)\right|^{2}\right]-20 \log _{10}\left(p_{r e f} \sqrt{2} R\right) \quad k R>>1
$$

In order to find the maximum noise on the ground outside the airport boundary, we need to construct a footprint grid based on the noise that was originally radiated from the engine exhaust nozzle. In practice, we only need to monitor the noise along the locus of points that run directly beneath the aircraft and along the sideline boundary of the airport as experience shows that this is where the point of maximum noise will occur. From Fig. 5 we see that any ground co-ordinate can be defined relative to the aircraft, in terms of the azimuthal and polar angles, $\psi$ and $\varphi$ and the aircraft height, $\mathrm{H}$. In addition, the aircraft climbs at an angle $\beta$ whilst experiencing an angle of attack $\alpha$. 


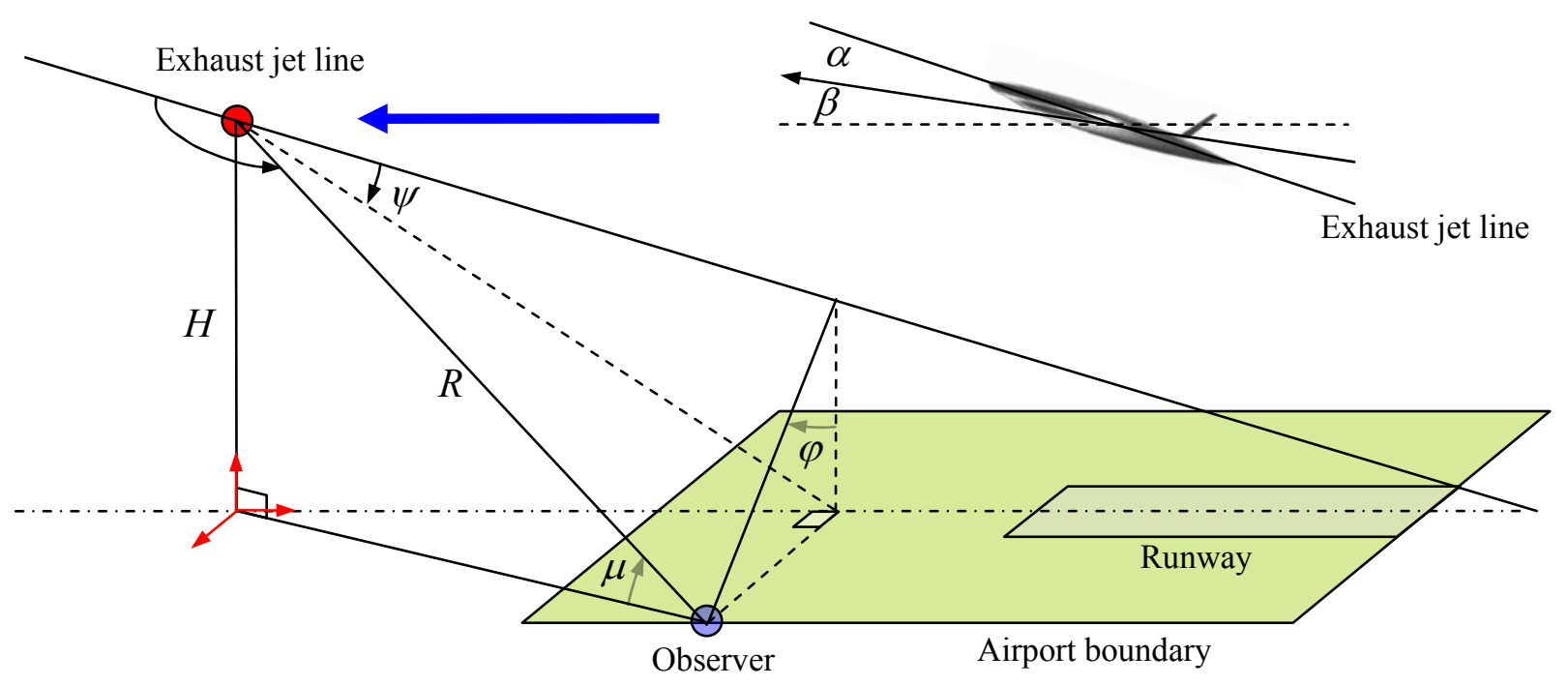

Figure 5. Defining the parameters required to evaluate the maximum observer noise outside of an airport boundary.

As well as spherical spreading, the noise levels that reach the observer at an actual airport will have been modified by atmospheric and lateral attenuation, ground reflection and Doppler shifting. By lateral attenuation, we are referring to the sum of all effects due to non-ideal conditions: the absorption of sound as it propagates over the porous surface that is the ground and any source installation effects. All but the last of these can be decoupled from the amplitude of the incident sound, which means that we can calculate them as corrections prior to the actual optimization. Atmospheric and lateral attenuation can be estimated using ESDU databases ${ }^{19,20}$. Atmospheric attenuations in this case are calculated for a uniform atmosphere at ground conditions. Lateral attenuation is estimated for a fuselage mounted engine as this is closer to the configuration of the Silent Aircraft. This is a function of the propagation distance and the elevation angle, $\mu$ for any third octave band. Ground reflection effects are accounted for by adding a further correction of $3 \mathrm{~dB}$ independent of the frequency. This is in line with recommendations made by Pierce ${ }^{21}$. Doppler corrections have to be omitted as inclusion would add an unacceptable amount of time to the optimization loop. Given that we are considering rearward propagating noise, the fact that we are working in the aircraft reference frame will mean that sound pressures would be expected to be lower anyway.

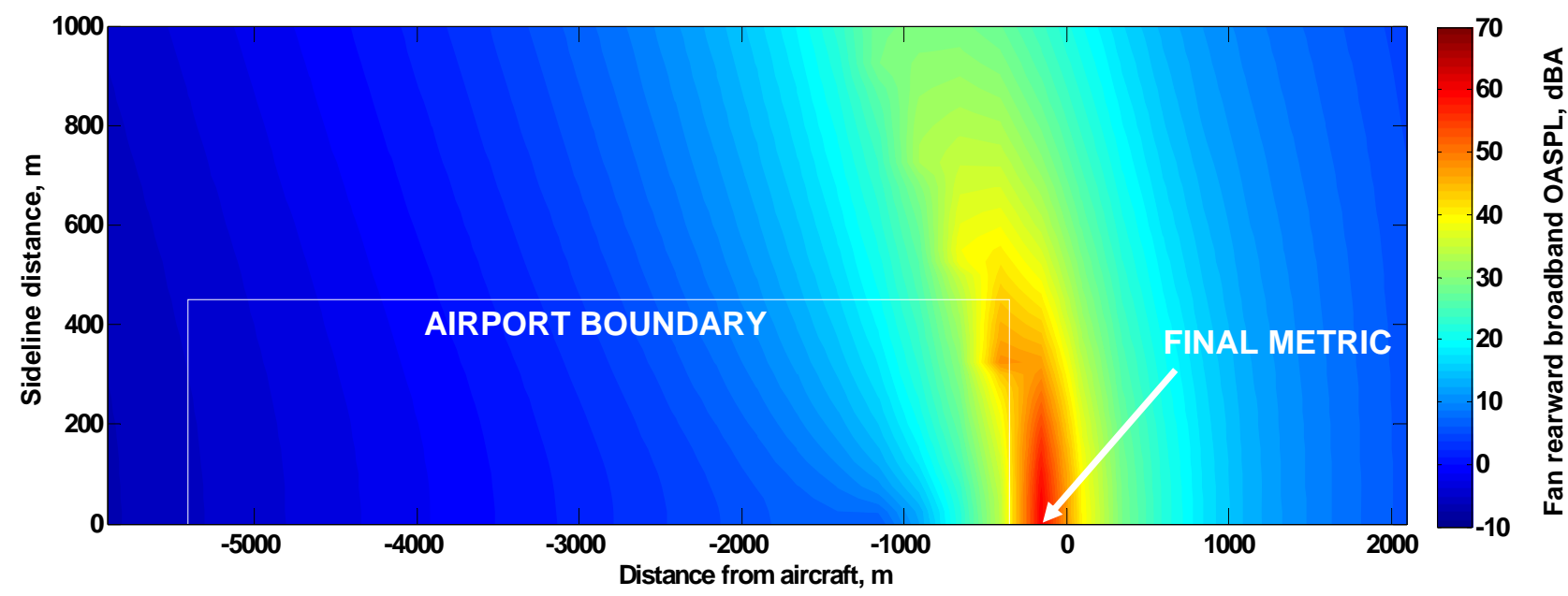

Figure 6. Fan rearward broadband footprint showing optimization metric for multiple liners in the mixed exhaust. 
The baseline airport used in this cost function was considered to be representative of the most restrictive of airports in London based on an extensive study ${ }^{22}$. The runway length was set at $3048 \mathrm{~m}$ with the airport boundary being a further kilometer at either end. The sideline distance to the boundary of $450 \mathrm{~m}$, was also deemed appropriate. Flight parameters for the flyover position have been extracted from the Silent Aircraft flight path at takeoff. Airport conditions are set to ISA $+12^{\circ} \mathrm{K}$ at $70 \%$ humidity, which is expected to give pessimistic results for $99 \%$ of operations. In the interest of brevity, we have not included any of the equations here as they are based on simple trigonometric relations. Should the reader wish to explore further, more detail has been given in a similar method by Crichton $^{23}$. A resulting high resolution noise footprint from the aircraft is shown in Fig. 6. Eq. (24) is used to radiate the sound onto a 100 feet radius hemisphere beneath the aircraft. The corrections are then added to determine the result on the ground for each third octave band. We integrate the values over frequency with an A-weighting before selecting the maximum SPL in dBA outside of the airport as our final metric. Clearly, this cost function could also be applied to the bypass duct if there were no mixed exhaust present as is the case for a lot of turbofan designs currently in service. One would merely have to adjust the modal directivity factors to account for the engine centre body $^{18}$.

\section{Results}

All optimizations have taken place on the aircraft flight path at the point that the aircraft cross the airport boundary. This is the critical condition for observer noise. At this point the aircraft is experiencing an angle of attack of $12.0^{\circ}$, whilst climbing at an angle of $3.1^{\circ}$ at a height of 308 meters. The Silent Aircraft is an interesting design problem as many of the installation parameters that would normally be fixed by the time it comes to designing the acoustic liners can still be modified as the overall goal is noise suppression. In particular, there was the option of modifying the engine nozzle lengths in order to meet the noise target. Consequently, it became useful to run multiple optimizations changing the overall length of the installation downstream of the fan and the relative lengths of the bypass versus the mixed exhaust to observe the benefit. The results are summarised in Fig. 7 where the final SAI design parameters are also indicated. The ratio of inner to outer radius in the annulus is 0.57 . One can see how much more effective the bypass liners appear to be at reducing the observer noise. The optimized liner parameters associated with this design for both the annular and cylindrical duct sections are detailed in Appendix B.
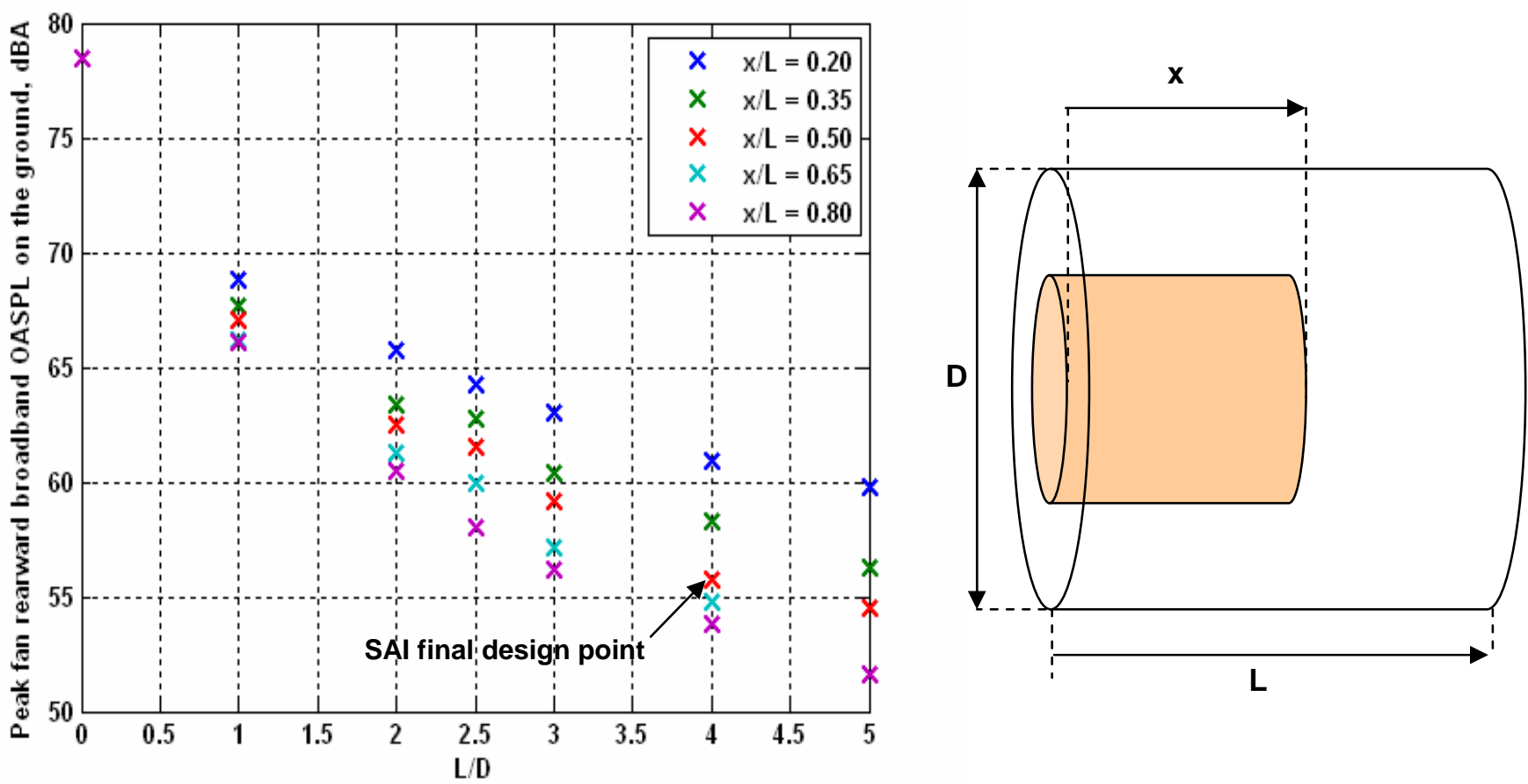

Figure 7. The effect on maximum observer noise of altering the annular and cylindrical liner lengths for the SAI design. 


\section{Acoustic Scattering}

\section{A. Model}

The approach that we have chosen to evaluate the scattered acoustic field is based on the work described earlier by McAlpine et $\mathrm{al}^{10}$. We consider a system of $\mathrm{d}$ liners placed next to one another as shown in the diagram below.

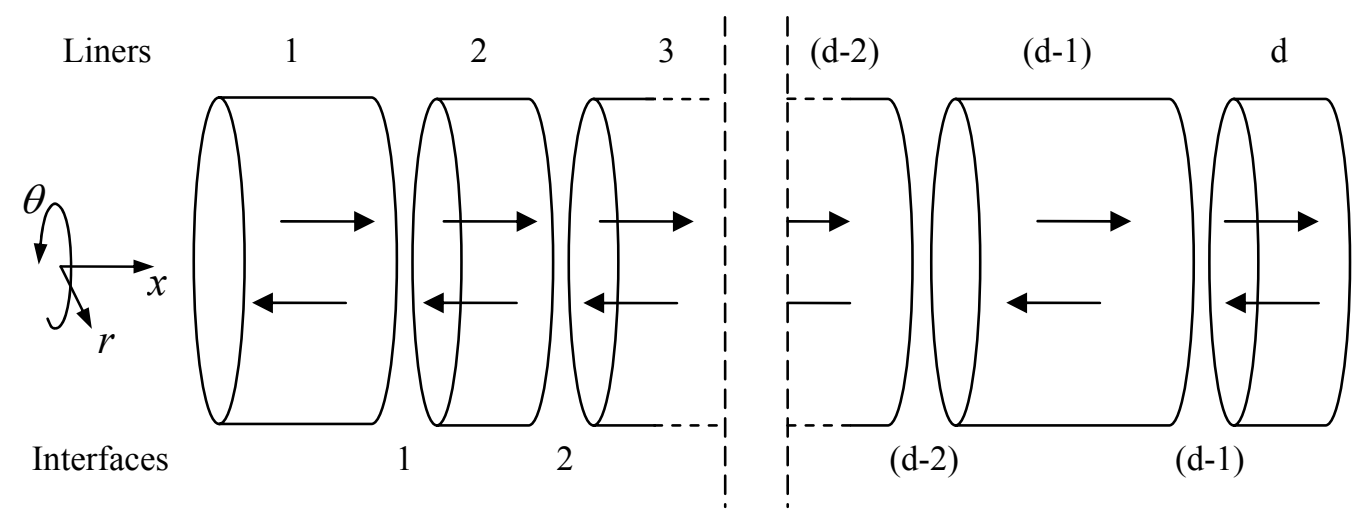

Figure 8. System of $d$ liners over which to evaluate acoustic scattering

At each interface we look to match the acoustic pressure and axial velocity, $u_{x}$, across the entire cross-section for either an annular or cylindrical duct. As the system has rotational symmetry, we can consider all the modes in each azimuthal order separately. The fundamental equations that are to be solved are Eqs. (25) and (26) where the pressure and axial velocity are summed over the radial modes only. The terms at the front of the integration are included so that the integral can be evaluated analytically to speed up the solution time. In addition, it allows us take advantage of the orthogonality of the Bessel Functions should we wish to insert rigid wall sections. The positive and negative superscripts denote that we are considering the pressures and velocities immediately after and before the liner junction.

$$
\begin{aligned}
& \int_{b}^{a} r \mathrm{X}_{n}\left(\kappa_{n} r\right)\left[\hat{p}_{n}^{d+1}\left(r, \theta, x_{d}^{+}\right)-\hat{p}_{n}^{d}\left(r, \theta, x_{d}^{-}\right)\right] \partial r=0 \\
& \int_{b}^{a} r \mathrm{X}_{n}\left(\kappa_{n} r\right)\left[\hat{u}_{x, n}^{d+1}\left(r, \theta, x_{d}^{+}\right)-\hat{u}_{x, n}^{d}\left(r, \theta, x_{d}^{-}\right)\right] \partial r=0
\end{aligned}
$$

The solution method involves the creation of $(d-1)$ transfer matrices, one for each interface. In the interests of brevity, the reader is directed towards the reference ${ }^{10}$ for the full details of how one may set up the full matrix equations. The work is based exclusively on cylindrical systems, however all the principles are applicable to an annulus. Clearly, there are an infinite number of radial modes for each value of $\mathrm{n}$ and therefore it becomes necessary to truncate the number at an appropriate point. In practice we find that as we include more modes the solution tends to converge rapidly at first with diminishing returns after that as one would expect. If one includes too many modes the matrix equations become ill-conditioned and fail to solve appropriately. This is because the routine is trying to allocate a very small amplitude to the highest radial modes. Consequently, there is a balance where one can extract the appropriate solution. The number of radial modes at which this threshold occurs can vary depending on how significantly the modes are scattered. If the modes are relatively unaffected by a system of liners, less modes are usually required to gain an acceptable solution. 


\section{B. Scattering Study}

For this paper, we shall restrict our study of the acoustic scattering to a cylindrical duct to help establish the principles behind broadband scattering. Future research is likely to contain results from annular geometries. We shall take the optimum liner design derived for the mixed exhaust on which to base our study (see Appendix B for details). Aside from the amount of energy entering the cylindrical duct, we will isolate the rest of the installation and its results from this study to improve transparency. The inlet condition is thus prescribed as equal energy per cut-on mode, whilst the downstream boundary condition at the end of the final liner is anechoic, in a similar way to McAlpine et $\mathrm{al}^{10}$. To ensure a satisfactory solution each time, we begin with only three additional radial modes and then solve the equations repeatedly whilst incrementing the number of modes until the solution converges as described in the previous section. This kind of scheme is also useful in automating the entire calculation for all azimuthal orders cut-on at a particular frequency. Third octave centre frequencies ranging from $400 \mathrm{~Hz} u p$ to $2 \mathrm{kHz}$ have been explored in this manner.

We shall begin by evaluating the scattering of the liners in their original order. Frequencies around $2 \mathrm{kHz}$ are potentially one of the most important to the observer, so we shall initially concentrate our attention here in a bid to simplify the analysis. It is instructive to consider the transfer of acoustic energy as we move along the duct to try and identify where the energy is being absorbed and/or amplified. For this we cannot use the analytical solution of Eq. (22) as the propagation is through a series of 'soft' walls. The integral in the acoustic power must be determined numerically as per Eq. (27). The frequencies at which each liner performs best and the corresponding length-todiameter ratios are displayed in Table 1 for convenience.

$$
W_{n m}^{ \pm}=\left|A_{n m}^{ \pm}\right|^{2} \Omega_{\mathrm{nm}}^{ \pm} \int_{0}^{a}\left|J_{n}\left(k_{r, n m}^{ \pm} r\right)\right|^{2} r \partial r
$$

Table 1. Key liner parameters for acoustic scattering.

\begin{tabular}{|c|c|c|}
\hline \multirow{2}{*}{ Liner } & $\begin{array}{c}\text { Peak operating frequency, } \\
\mathrm{Hz}\end{array}$ & Segment L/D \\
\hline 1 & 510 & 0.102 \\
\hline 2 & 1350 & 0.133 \\
\hline 3 & 1570 & 0.188 \\
\hline 4 & 2250 & 0.863 \\
\hline 5 & 2760 & 0.279 \\
\hline 6 & 3480 & 0.069 \\
\hline 7 & 3580 & 0.366 \\
\hline
\end{tabular}

Two plots showing residual acoustic energy in radial modes are presented in Fig. 9 for the liners in their original order 1-7 for non-spinning modes and an azimuthal order of ten. We have only considered modes that travel downstream as our aim is to prevent energy reaching the end of the system, which includes the possibility of reflection from the interfaces. Duct positions 0 and 7 refer to energies at the inlet and outlet respectively. The - and + symbols correspond to immediately upstream and downstream of the liner junction, meaning that they allow one to see the isolated effect of the interface. Each bar is given in decibels; the stacking of modes provides a convenient way of viewing the effects on all modes together. However, this does mean that the overall height becomes meaningless and therefore this is not a tool for comparing different designs (absolute values on the vertical axis are consequently omitted). In the plot we include one cut-off mode directly and then combine the energy in the higher radial modes into a single data point. One of the key aspects of the results is the amount of energy that is transferred to the cut-off modes by the junctions. Unless the following liner segment is very short, the energy in these modes is likely to be virtually eradicated by the liner before the next junction. To transfer energy to these modes is therefore an efficient attenuation mechanism. 

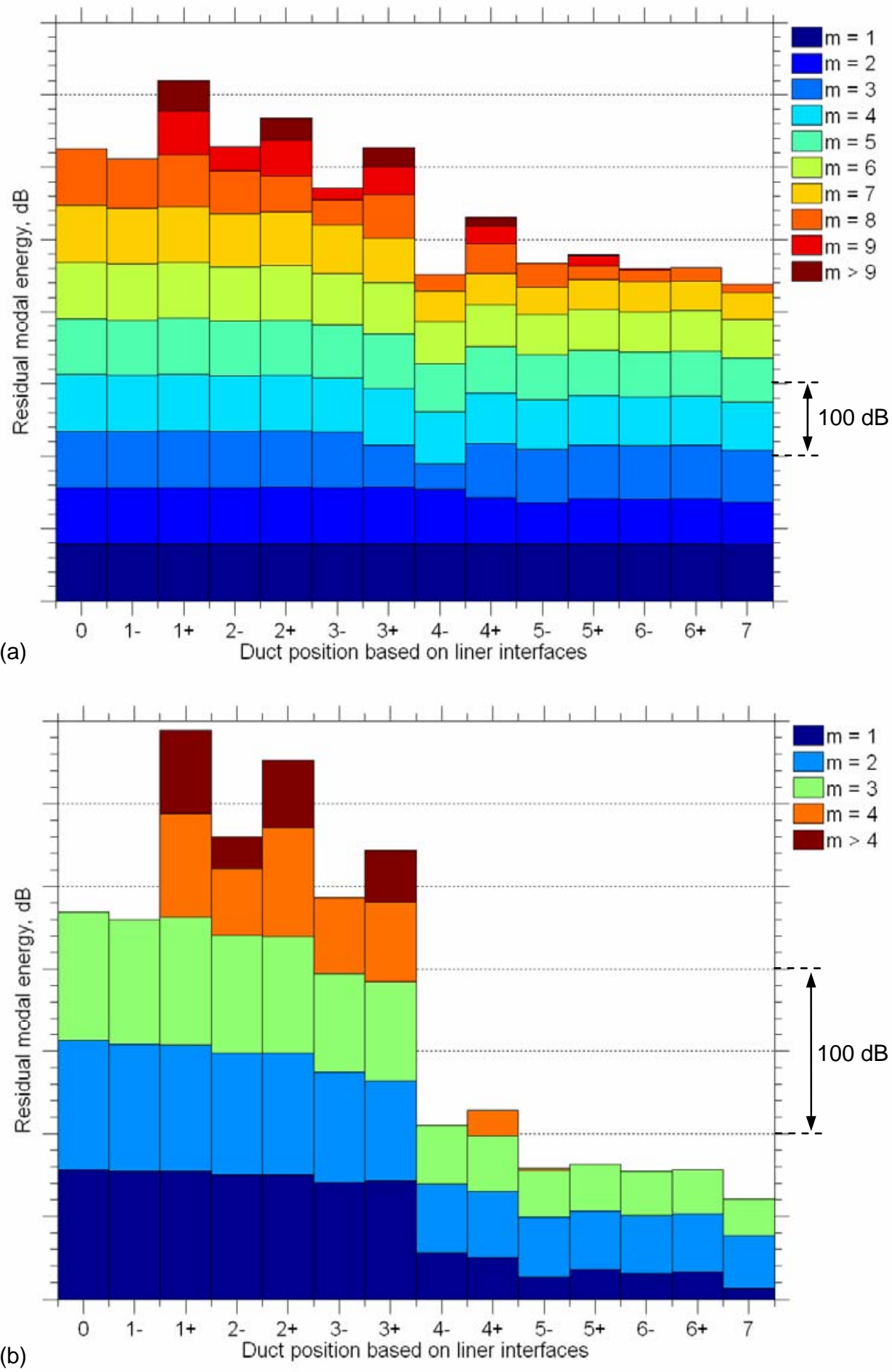

Figure 9. Residual energy in radial modes travelling downstream for azimuthal orders $(a) n=0$, and $(b) n=$ 10 for frequency $2 \mathrm{kHz}$ for the original liner order. 
Initially, we observe that the largest single attenuation is due to the action of the fourth liner. This is not surprising as it is the one tuned most closely to the frequency we are considering and its length is significantly longer than the other liners in the system. The effect on the plane wave is minimal; modes of larger axial wavelength do not 'see' as much of the interface and consequently will often pass through virtually unaffected. The two liners that have very similar peak operating frequencies (and therefore similar wall impedances) only have a very small scattering effect across the sixth interface as one might expect. Further interrogation of the results indicates that in the case of an apparent amplification across a liner interface, the energy entering the junction is conserved by the waves travelling in the upstream direction. It becomes difficult to make an overall judgement based on these plots since there are many azimuthal orders to be considered. So, in order to assess the overall behavior, we compute the superposition across all azimuthal orders. The relevant plot for the liner that is being discussed currently is given as Fig. 10. In general, scattering at the liner interfaces fails to provide a benefit in attenuation and the overall attenuation value for this system is $15.3 \mathrm{~dB}$ compared to $19.6 \mathrm{~dB}$ for the case when scattering is neglected.

The question that now becomes pressing is whether we can reduce the amount of power transmitted by reordering the liners. Analysis of several different solutions at the same frequency appears to suggest that the relative absorption or amplification effects obtained by placing two liners next to one another is very similar independent of where they are placed in the sequence. The difference being the amount of energy present in the modes incident on that particular section. This suggests that if one arranges the liners to minimize as much energy as possible early on we can negate any undesirable scattering effects. Clearly, this theory implies that liner number four should be placed earlier on in the system. We can go further and place liners 3 and 5 second and third as both are likely to be able to attenuate a $2 \mathrm{kHz}$ signal somewhat. This leads to Liner System 1 as listed in Table 2. The residual radial energy plot for this liner order is shown in Fig. 11(a). The energy in the system is attenuated by the upstream liners, thus reducing the adverse effects of the scattering seen for the original liner order. If we apply this logic to the original system, we can see that it was not a particularly good solution. The first segment is designed to attenuate low frequencies and the harmonic nature of the liner response (see Figure 33 ) dictates that attenuation will be very poor at around four times its peak operating frequency $(\sim 2 \mathrm{kHz})$. Therefore, it gives no benefit and combines with the second liner to form an amplifying scatterer at $2 \mathrm{kHz}$. Energy removal rates improve as we move downstream through the liners but it appears that it is hard for the liner system to recover from the poor performance in the first two liners. It is possible to make the situation worst by moving the most effective liner (Liner 4) further downstream and replacing it with the smallest segment that also performs poorly at $2 \mathrm{kHz}$, as in Liner System 2.

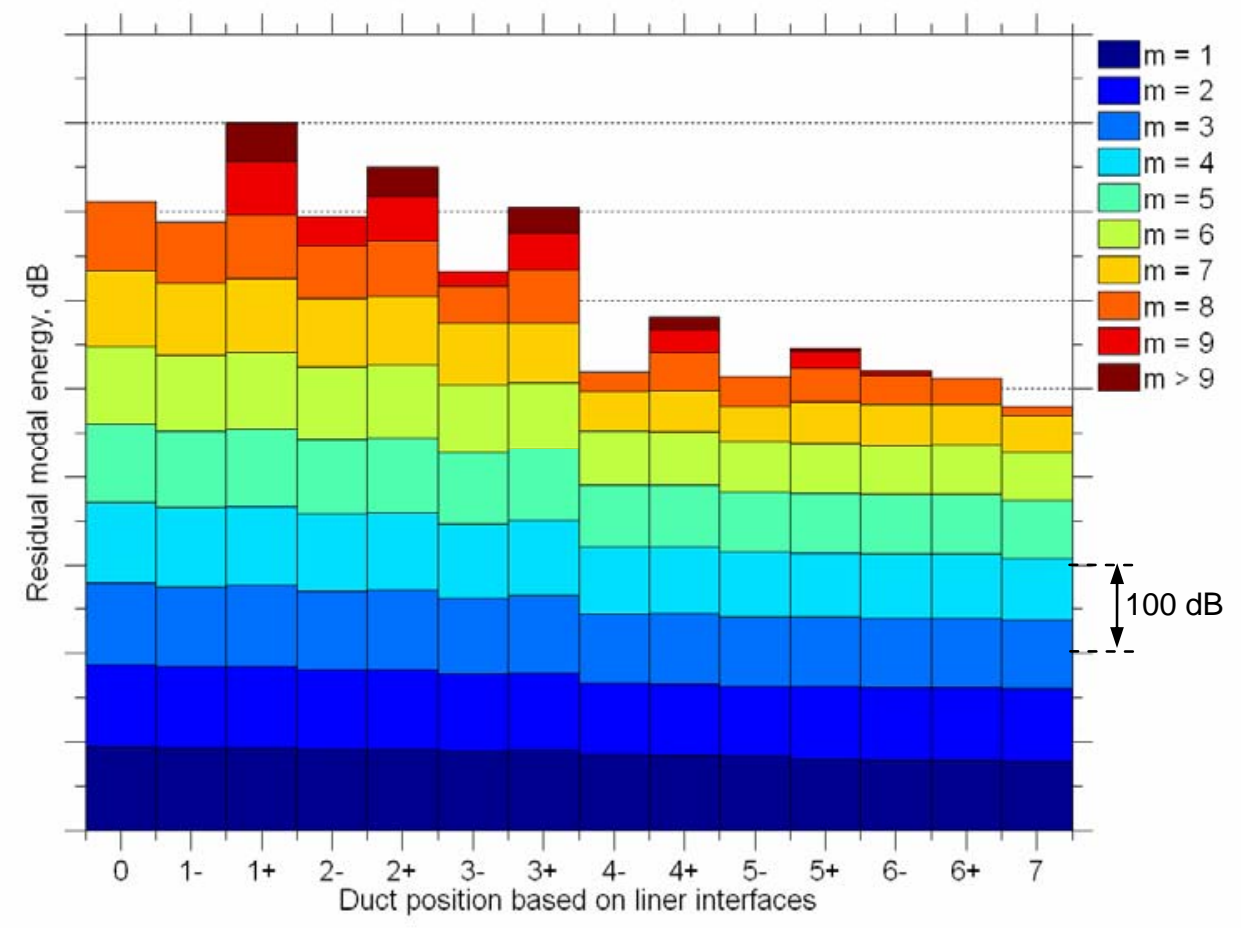

Figure 10. Residual energy in radial modes summed over azimuthal order for the original liner order. 
Table 2. Liner orders for the three systems presented and overall performance at $2 \mathrm{kHz}$.

\begin{tabular}{|c|c|c|c|c|c|c|c|c|}
\hline Liner system & $1^{\text {st }}$ & $2^{\text {nd }}$ & $3^{\text {rd }}$ & $4^{\text {th }}$ & $5^{\text {th }}$ & $6^{\text {th }}$ & $7^{\text {th }}$ & $\begin{array}{c}\text { Overall PWL } \\
\text { attenuation, dB }\end{array}$ \\
\hline Original & 1 & 2 & 3 & 4 & 5 & 6 & 7 & 15.35 \\
\hline 1 & 4 & 3 & 5 & 1 & 2 & 6 & 7 & 18.28 \\
\hline 2 & 1 & 2 & 3 & 6 & 5 & 4 & 7 & 14.76 \\
\hline
\end{tabular}
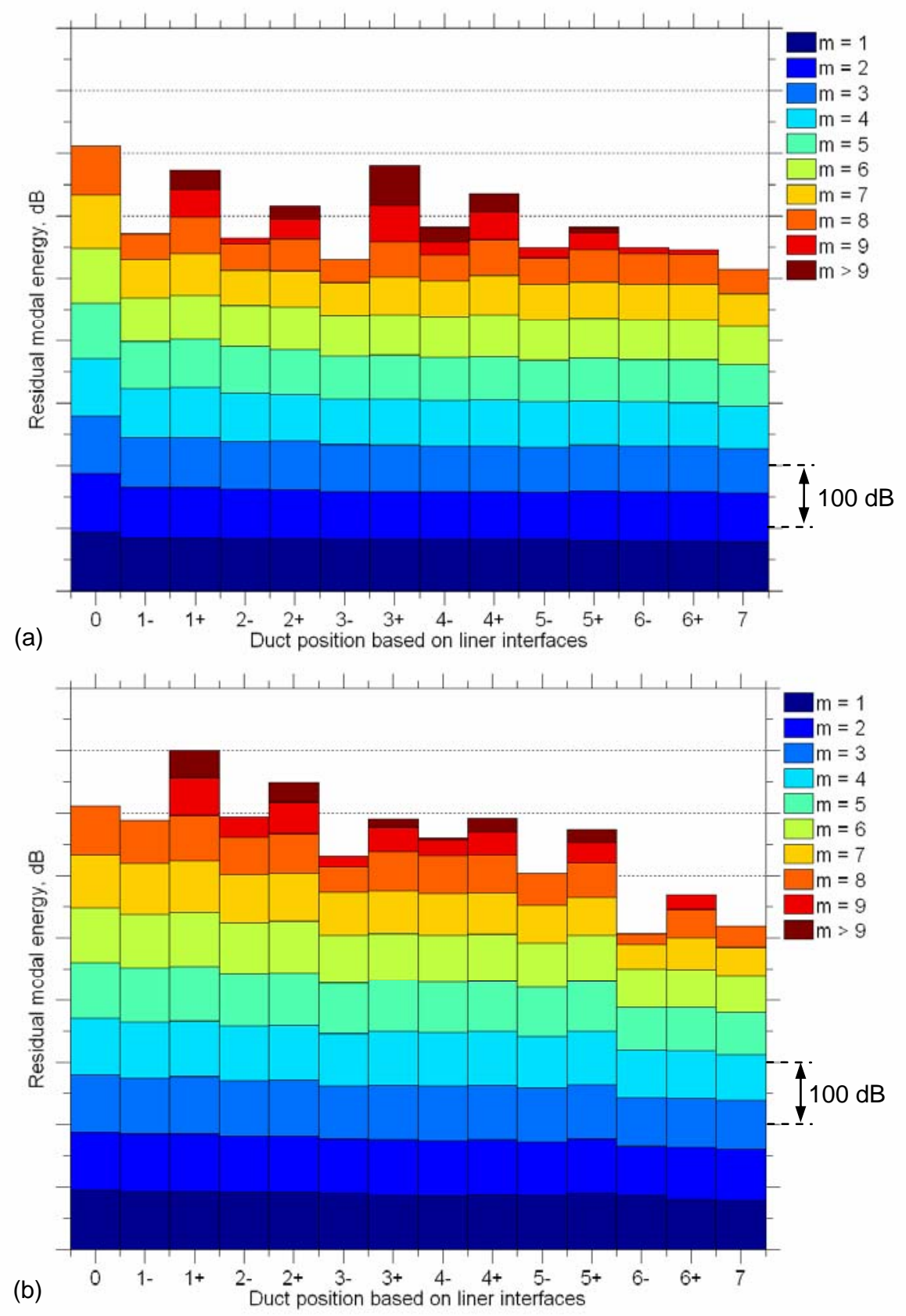

Figure 11. Residual energy in radial modes summed over azimuthal order for rearranged liner orders (a) 1 and (b) 2 at frequency $2 \mathrm{kHz}$. 
The effect of Liner System 2 is shown in Fig. 11(b). This liner has failed to transfer as much energy from the earlier radial modes by the end of the final liner meaning that the overall attenuation has fallen to $14.8 \mathrm{~dB}$. The apparent ability to order the liners based on their performance when placed in a two liner system raises the possibility of developing a method based on transfer functions for adjacent liners. If this is the case, it would mean that the whole set of permutations for $d$ liners could be covered based on $d(d-1)$ calculations, which for a number of liners greater than 3 represents an increasingly large possible saving in computation time over $d$ factorial calculations. This is beyond the scope of this paper but may be an area of future interest.

Of course, this is not the end of the problem as broadband noise covers a much wider spectrum than simply that around $2 \mathrm{kHz}$. The figure below displays the performance of the liners presented in Table 2 over the third octave centre frequencies from $400 \mathrm{~Hz}$ to $2 \mathrm{kHz}$. This includes a comparison with the result when scattering is neglected. Based on the results for this investigation, we can achieve a similar performance to the case when scattering is neglected at the frequencies that are most important to the observer. It may be possible to gain a slight improvement across certain frequency bands; however there is the possibility of losing a significant amount of the attenuation if the liners are ordered in a naive manner. Given that it becomes possible to achieve something close to the no scatter result with relatively little effort, the liner optimization models for Sections II and III would appear to be a valid way of designing the acoustic treatment for a mixed exhaust style aeroengine. Furthermore, it follows that scattering (and therefore liner order) will become more important if only shorter liner segments are used. Conventional turbofans will rarely have the luxury of such a long mixed flow exhaust and are therefore likely to be more severely affected.

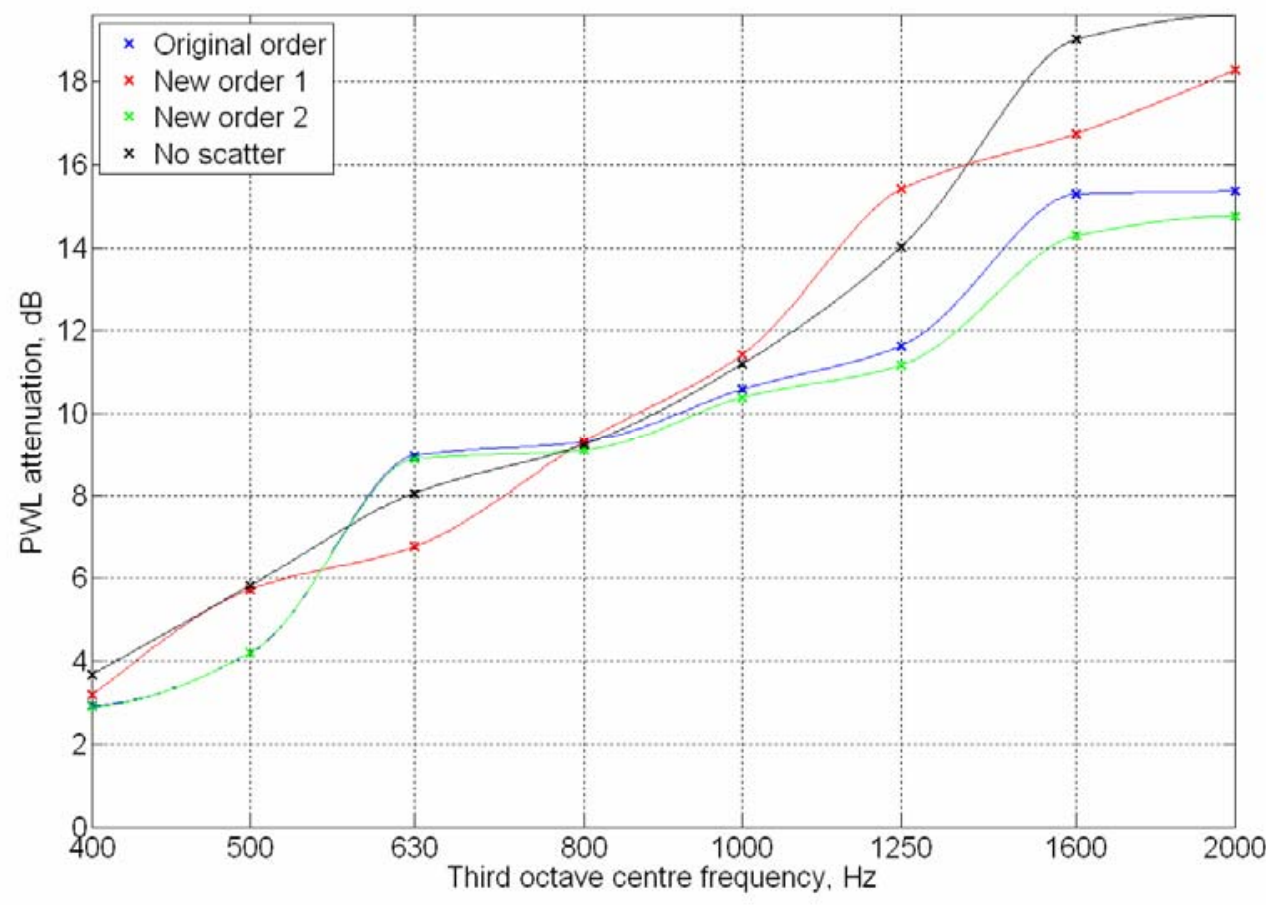

Figure 12. Performance of the three liner designs specified in Table 2 in comparison with the no scatter solution for the third octave centre frequencies from $400 \mathrm{~Hz}$ to $2 \mathrm{kHz}$.

\section{Conclusion}

A method for optimizing the acoustic treatment for the bypass duct and mixed exhaust of an aeroengine has been outlined. It is designed to provide the maximum possible noise benefit to the observer by use of intelligent cost functions. It has been successfully applied to the Silent Aircraft concept design in order to meet the noise target of 
$57 \mathrm{dBA}$ as the aircraft passes over the airport boundary. The significant improvement in performance per unit length of an annular liner compared to a cylindrical one has been exploited in order to achieve this. The optimization procedure requires one to assume that acoustic scattering between axial liner segments can be neglected. We have tested this hypothesis by evaluating the scattering for the original liner configuration and for other permutations of the same liners using the method of weighted residuals. For the optimized liner design in the mixed exhaust, it was found that scattering was likely to lead to a reduction in sound power attenuation. At $2 \mathrm{kHz}$, we demonstrated that it was possible for the system to yield $4.8 \mathrm{~dB}$ lower attenuation than the case in which scattering is neglected if the liners are arranged in certain orders. The results suggest that to obtain better overall performance at a particular frequency, one should eradicate as much of the noise energy as possible in the upstream segments of liner to reduce the detrimental effects of scattering as much as possible. Poorer designs will have a short segment at the beginning that is not well 'tuned' to the frequency of interest. This will often result in making the task more difficult for the remaining liners downstream. By intelligent positioning of acoustic absorbers it is possible to achieve a performance comparable to the case in which scattering is neglected. Consequently, the earlier assumption that facilitates the system optimization is a valid one to make at the high level design stage for the SAI mixed exhaust aeroengine. Existing (or more incremental) mixed exhaust designs are likely to be constrained to shorter liner segments than the SAI design. Therefore, it follows that scattering and liner order will become more influential in these cases.

\section{Appendix}

\section{A. Convergence to Annular Eigenvalues from Cylindrical Ones}

Due to the increased complexity of the annular eigenequation, it often becomes easier to obtain the low order solutions by computing the cylindrical ones first. A proof that the annular solution converges to the cylindrical one as $\mathrm{b} / \mathrm{a}$ tends to zero is given here:

To begin, we note that for small argument ${ }^{16}$, the Bessel functions of the First and Second Kind become:

$$
\begin{gathered}
J_{n}(x) \sim \frac{[x / 2]^{n}}{\Gamma(n+1)} \\
Y_{0}(x) \sim \frac{2}{\pi} \ln (x) \\
Y_{n}(x) \sim-\frac{1}{\pi} \Gamma(n)[x / 2]^{-n}
\end{gathered}
$$

Consider each of the four main components that form Eq. (10) separately:

$$
\begin{gathered}
\left(n Z_{1}-C_{n m}^{ \pm}\right) J_{n}\left(k_{r, n m}^{ \pm} a\right)-k_{r, n m}^{ \pm} a Z_{1} J_{n+1}\left(k_{r, n m}^{ \pm} a\right) \\
\left(n Z_{2}-C_{n m}^{ \pm} b / a\right) Y_{n}\left(k_{r, n m}^{ \pm} b\right)-k_{r, n m}^{ \pm} b Z_{2} Y_{n+1}\left(k_{r, n m}^{ \pm} b\right) \\
\left(n Z_{1}-C_{n m}^{ \pm}\right) Y_{n}\left(k_{r, n m}^{ \pm} a\right)-k_{r, n m}^{ \pm} a Z_{1} Y_{n+1}\left(k_{r, n m}^{ \pm} a\right) \\
\left(n Z_{2}-C_{n m}^{ \pm} b / a\right) J_{n}\left(k_{r, n m}^{ \pm} b\right)-k_{r, n m}^{ \pm} b Z_{2} J_{n+1}\left(k_{r, n m}^{ \pm} b\right)
\end{gathered}
$$


Now, let the radial wavenumbers in each expression be the equivalent cylindrical ones. Eq. (31) is the cylindrical eigenequation (Eq. (7)) and will be equal to zero by definition. Eq. (32) can be simplified as b/a tends to zero into:

$$
\begin{array}{ll}
\frac{\left(\mathrm{k}_{\mathrm{r}, \mathrm{nm}}^{ \pm} \mathrm{b} / 2\right)^{-\mathrm{n}}}{\pi}\left[n Z_{2}+C b / a\right] \Gamma(n) & n \neq 0 \\
\frac{2}{\pi}\left[Z_{2}-C(b / a) \ln \left(k_{r, n m}^{ \pm} b\right)\right] & n=0
\end{array}
$$

Eq. (33) we will see, requires no simplification as it will yield a finite complex number while Eq. (34) can be rewritten as Eq. (37).

$$
\frac{\left(\mathrm{k}_{\mathrm{r}, \mathrm{nm}}^{ \pm} \mathrm{b} / 2\right)^{\mathrm{n}}}{\Gamma(\mathrm{n}+1)}\left[n Z_{2}-C b / a-\left(k_{r, n m}^{ \pm} b\right)^{2} Z_{2} / 2(n+1)\right]
$$

If we reconstruct the full eigenequation we can write:

$$
\begin{aligned}
& \frac{n \Gamma^{2}(n)}{\pi}\left[\left(n Z_{1}-C_{n m}^{ \pm}\right) J_{n}\left(k_{r, n m}^{ \pm} a\right)-k_{r, n m}^{ \pm} a Z_{1} J_{n+1}\left(k_{r, n m}^{ \pm} a\right)\right]\left[n Z_{2}+C b / a\right]-\quad n \neq 0 \\
& \left(\mathrm{k}_{\mathrm{r}, n \mathrm{~m}}^{ \pm} \mathrm{b} / 2\right)^{2 \mathrm{n}}\left[\left(n Z_{1}-C_{n m}^{ \pm}\right) Y_{n}\left(k_{r, n m}^{ \pm} a\right)-k_{r, n m}^{ \pm} a Z_{1} Y_{n+1}\left(k_{r, n m}^{ \pm} a\right)\right]\left[n Z_{2}-C b / a-\left(k_{r, n m}^{ \pm} b\right)^{2} Z_{2} / 2(n+1)\right]=0 \\
& \frac{4}{\pi}\left[-C_{0 m}^{ \pm} J_{0}\left(k_{r, 0 m}^{ \pm} a\right)-k_{r, 0 m}^{ \pm} a Z_{1} J_{1}\left(k_{r, 0 m}^{ \pm} a\right)\right]\left[Z_{2}-C(b / a) \ln \left(k_{r, 0 m}^{ \pm} b\right)\right]- \\
& \mathrm{k}_{\mathrm{r}, 0 \mathrm{~m}}^{ \pm} \mathrm{b}\left[-C_{0 m}^{ \pm} Y_{0}\left(k_{r, 0 m}^{ \pm} a\right)-k_{r, 0 m}^{ \pm} a Z_{1} Y_{1}\left(k_{r, 0 m}^{ \pm} a\right)\right]\left[-C b / a-\left(k_{r, 0 m}^{ \pm} b\right)^{2} Z_{2} / 2\right]=0
\end{aligned}
$$

In addition it can be shown that:

$$
\lim _{b / a \rightarrow 0}(b / a) \ln \left(k_{r, 0 m}^{ \pm} b\right)=0
$$

Therefore, we obtain the final result for all cases that:

$$
\lim _{b / a \rightarrow 0}\left(\text { Eq.(10)) }=\left(n Z_{1}-C_{n m}^{ \pm}\right) J_{n}\left(k_{r, n m}^{ \pm} a\right)-k_{r, n m}^{ \pm} a Z_{1} J_{n+1}\left(k_{r, n m}^{ \pm} a\right)\right.
$$

Hence, the annular eigenvalues converge to the cylindrical ones at low radii ratios. 


\section{B. Optimized Liner Parameters}

This appendix contains the finer details of the different segments from the multiple liner optimization exercise for both the annular and cylindrical liner sets.

Table 3. Multiple liner parameters for the Silent Aircraft bypass duct.

\begin{tabular}{|c|c|c|c|c|c|c|c|c|c|c|c|}
\hline Segment & 1 & 2 & 3 & 4 & 5 & 6 & 7 & 8 & 9 & 10 & 11 \\
\hline Cell depth, mm & 202.6 & 180.6 & 143.4 & 120.7 & 95.86 & 90.50 & 57.10 & 48.05 & 22.73 & 10.15 & 9.05 \\
\hline Sheet porosity, \% & 10.01 & 16.00 & 9.98 & 15.13 & 9.94 & 12.98 & 13.31 & 15.07 & 12.88 & 10.13 & 10.03 \\
\hline $\begin{array}{c}\text { Length-to- } \\
\text { diameter ratio }\end{array}$ & 0.178 & 0.205 & 0.341 & 0.254 & 0.169 & 0.141 & 0.191 & 0.176 & 0.102 & 0.144 & 0.099 \\
\hline
\end{tabular}

Table 4. Multiple liner parameters for the Silent Aircraft mixed exhaust duct.

\begin{tabular}{|c|c|c|c|c|c|c|c|}
\hline Segment & 1 & 2 & 3 & 4 & 5 & 6 & 7 \\
\hline Cell depth, mm & 151.9 & 50.89 & 42.82 & 30.31 & 24.08 & 19.13 & 18.06 \\
\hline Sheet porosity, \% & 15.99 & 11.18 & 11.18 & 11.28 & 11.39 & 11.70 & 11.81 \\
\hline $\begin{array}{c}\text { Length-to- } \\
\text { diameter ratio }\end{array}$ & 0.102 & 0.133 & 0.188 & 0.863 & 0.279 & 0.069 & 0.366 \\
\hline
\end{tabular}

\section{Acknowledgments}

The authors would like to thank the Cambridge-MIT Institute and the Engineering and Physical Sciences Research Council for funding the research. Prof Jeremy Astley and Dr. Gwénaël Gabard at ISVR are acknowledged for allowing us the use of their Matlab routines to evaluate the modal directivity patterns for a semi-infinite duct. Finally, we would like to thank Daniel Crichton for allowing access to his Matlab routines that manipulate the output from the ESDU propagation software listed in this paper.

\section{References}

${ }^{1}$ Chapman, C. J., "Sound Radiation from a Cylindrical Duct. Part 1: Ray Structure of the Duct Modes and of the External Field," Journal of Fluid Mechanics, Vol. 281, No. 1, 1994, pp. 293-311.

${ }^{2}$ Rienstra, S. W., "A Classification of Duct Modes Based on Surface Waves," Wave motion, Vol. 37, 2002, pp. 119-135.

${ }^{3}$ Law, T. R. and Dowling, A. P., "Optimization of Traditional and Blown Liners for a Silent Aircraft," AIAA-2006-2525, 12th AIAA/CEAS Aeroacoustics Conference (27th AIAA Aeroacoustics Conference), Cambridge, MA, 2006.

${ }^{4}$ Ko, S. H., "Sound Attenuation in Acoustically Lined Circular Ducts in the Presence of Uniform Flow and Shear Flow," Journal of Sound and Vibration, Vol. 22, No. 2, 1972, pp. 193-210.

${ }^{5}$ ESDU, "The Acoustic Attenuation of Absorbent Linings in Cylindrical Flow Ducts," Item 00012, 2000.

${ }^{6}$ Astley, R. J. and Eversman, W., "A Finite Element Formulation of the Eigenvalue Problem in Lined Ducts with Flow," Journal of Sound and Vibration, Vol. 65, No. 1, 1979, pp. 61-74.

${ }^{7}$ Astley, R. J. and Eversman, W., "Wave Envelope and Infinite Element Schemes for Fan Noise Radiation from Turbofan Inlets," Journal of the Acoustical Society of America, Vol. 22, No. 12, 1984, pp. 1719-1726. 
${ }^{8}$ McAlpine, A. and Wright, M. C. M., "Acoustic Scattering by a Spliced Turbofan Inlet Duct Liner at Supersonic Fan Speeds," Journal of Sound and Vibration, Vol. 292, 2006, pp. 911-934.

${ }^{9}$ Unruh, J. F., "Finite Length Tuning for Low-Frequency Lining Design," Journal of Sound and Vibration, Vol. 45, No. 1, 1976, pp. 5-14.

${ }^{10}$ McAlpine, A., Astley, R. J., Hii, V. J. T., Baker, N. J., and Kempton, A. J., "Acoustic Scattering by an Axial-Segmented Turbofan Inlet Duct Liner at Supersonic Fanspeeds," Journal of Sound and Vibration, Vol. 294, 2006, pp. 780-806.

${ }^{11}$ Cummings, A., "High Frequency Ray Acoustics Models for Duct Silencers," Journal of Sound and Vibration, Vol. 221 , No. 4, 1999, pp. 681-708.

${ }^{12}$ Tyler, J. M. and Sofrin, T. G., "Axial Flow Compressor Noise Studies," Transactions of the Society of Automotive Engineers, Vol. 70, 1962, pp. 309-332.

${ }^{13}$ Motsinger, R. E. and Kraft, R. E., "Aeroacoustics of Flight Vehicles: Theory and Practice, Volume 2: Noise Control," NASA RP-1258, 1991, pp. 165-206.

${ }^{14}$ Guess, A. W., "Calculation of Perforated Plate Liner Parameters from Specified Acoustic Resistance and Reactance," Journal of Sound and Vibration, Vol. 40, No. 1, 1975, pp. 119-137.

${ }^{15}$ Morfey, C. L., "Sound Transmission and Generation in Ducts with Flow," Journal of Sound and Vibration, Vol. 14, No. 1, 1971, pp. 37-55.

${ }^{16}$ Abramovich, M. and Stegun, I. A., Handbook of Mathematical Functions, 9th ed., Dover, New York, 1970.

${ }^{17}$ Munt, R. M., "The Interaction of Sound with a Subsonic Jet Issuing from a Semi Infinite Cylindrical Pipe," Journal of Fluid Mechanics, Vol. 83, No. 4, 1977, pp. 609-640.

${ }^{18}$ Gabard, G. and Astley, R. J., "Theoretical Model for Sound Radiation from Annular Jet Pipes: Farfield and Nearfield Predictions," Journal of Fluid Mechanics, Vol. 549, No. 1, 2006, pp. 315-341.

${ }^{19}$ ESDU, "Evaluation of the Attenuation of Sound in a Uniform Atmosphere," Item 78002, 1977.

${ }^{20}$ ESDU, "Estimation of Lateral Attenuation of Air-to-Ground Jet or Turbofan Aircraft Noise in One-Third Octave Bands," Item 82027, 1982.

${ }^{21}$ Pierce, A. D., "Acoustics: An Introduction to its Physic Principles and Applications," 2nd ed., The Acoustical Society of America, New York, 1989, pp. 104-106.

${ }^{22}$ Tam, R. and Reynolds, T., "Take-Off Requirements for the SAI Silent Aircraft," CMI, 2006.

${ }^{23}$ Crichton, D., Fan Design and Operation for Ultra Low Noise, Ph. D. Thesis, Department of Engineering, University of Cambridge, 2007. 